The Internet Movie Database (a.k.a. Cardiff's Movie Database). Access: http://www.cm.cf.ac.uk/ Movies/moviequery (also available via e-mail: send subject "help" to movie@ ibmpcug.co.uk).

The Internet Movie Database illustrates the great potential of the Internet. Since 1989 volunteers under the direction of Col Needham have contributed to and coordinated additions and corrections from the Internet community to create a database that now lists over 40,000 movie, documentary, and television series titles and over 500,000 of the people credited with making them.

The Internet Movie Database includes movies produced in every part of the world and television series produced in the United States, Canada, and the United Kingdom. The movies range from silents to films currently in production. The movies tend to be mainstream, listing very little in the way of experimental films, lesser-known documentaries, or pornography. The database is usually updated weekly.

Searching the database is as easy as point and click. In addition to title and name searches, the searcher can generate lists of films by certificate (age rating), country of origin, production company, or genre (as yet uncontrolled descriptors). The database provides a search form for character names, plot summaries, selected quotations, locations, soundtracks, year of release, and rating (a grade from one to ten voted on by the Internet community).

The Internet Movie Database has three main drawbacks. First, the database offers no boolean search capabilities. Second, the database is far from complete. Fewer than one in ten movies contain a complete physical description and summary, genre classifications, cast and credits, or age rating. Fewer than one in 14 provide links to the good, quality reviews archived in the rec.arts.movies.reviews Usenet newsgroup. Fewer than one in 20 include biographies. Finally, because the information comes directly

Sara Amato is automated systems librarian al Central Washington lniversity; samato@taboma.cum.edu from Internet users, the quality and authority of records vary.

Because searching is only possible by one clata element at a time, the Internet Movie Database is best used to produce filmographies. Although all of the search inclexes listed above are available, users who wish to view a film or individual's credit list will benefit the most. While not yet authoritative or complete enough to take the place of the CI)-ROM movie databases or the multivolume print compilations, the Internet Movie Database is highly recommended. Besides, what other film resource allows reaclers to contribute ratings and corrections or submit their own entries?-Mark Emmons, Occidental College, e-mail: mee@oxy.edu

\section{Federal Communications Commission}

Gopher. Access: gopher://gopher.fcc.gov; http://www.fcc.gov; ftp//ftp.fcc.gov

The Federal Communications Commission (FCC) gopher could be very useful to anyone involved with communications, especially those who have to keep up with its latest regulations. The gopher houses much valuable information, but using it is a daunting experience without knowledge of the FCC's general structure.

The main gopher menu includes 31 different categories. What it desperately needs is a README file. Files ending in .wp (WordPerfect readable) fill one's screen with computer language, and compressed files (.zip) have to be "unzipped." Universally available WAIS searching is never explained and thus is rendered virtually useless.

Only via the FCC frp site README file was I able to decipher bureau codes used in various submenus. According to an FCC official, the site came up in February 1994, is still under construction, and will be upgraded as soon as possible. I encountered some problems connecting to the site, although I received a swift return telephone call from someone at the FCC and via e-mail from cjboyer@uci.edu (whose 
address I found at the FCC ftp site). The somewhat helpful subdirectory "index.txt" unfortunately moved from near the beginning to near the end of the submenus on subsequent visits.

The Daily Common Carrier items are very current, arranged in reverse chronological order, and go back through 1994. After wading through too many subdirectories, one finds valuable information such as tariff transmittal schedules, public notices, news releases, FCC studies, complete texts or ordering information, and notices and requests for comments on proposed rule-making. The Daily Digest section is less well organized with the dates inexplicably mixed up and a lot of material repeated from elsewhere. The Daily Business/Cable subdirectory has an enormous 618 entries.

Other useful subdirectories include News Releases, Events, Panel Discussion, Speeches, and Forms. Less useful were Phone Books (zipped), GN-Docs (a very technical WAIS setup guide), and Informal. International and Fees were repeatedly inaccessible. Unfortunately, spelling mistakes abound throughout and too much information is repeated.

The FCC gopher has valuable, current information but it needs to be more clearly and simply organized to make it easier for the public and librarians to use.-Elaine Hoffman, State University of New York at Stony Brook, e-mail: EHOFFMAN@CCMAIL.SUNYSB.EDU

\section{The Nine Planets: A Multimedia Tour of the Solar System. Access: http://seds.lpl. arizona.edu/billa/tnp. Owner and contact: Bill Arnett (http://seds.lpl.arizona.edu/billa/ arnett.html)}

The Nine Planets is a hypertext-based tour of text and images about the solar system. Designed for general audiences, the tour considers each of the objects in our solar system: the Sun, planets, their satellites, asteroids, and comets. Users browse among approximately 65 HTML pages that provide inline images of the objects, discursive text, charts of physical data, and links to a variety of images and animations.

Any technical vocabulary is linked to a glossary which defines the terms in relatively simple language.

Images are plentiful throughout the tour. They are taken from or point to a variety of sources, mostly at NASA and other government sites. In fact, many of the images come from two similar World Wide Web sources: Welcome to the Planets (http://stardust.jpl.nasa.gov/planets/) and Views of the Solar System (http:// www.c3.lanl.gov/ cjhamil/SolarSystem/ homepage.html). Arnett's tour is differentiated by its fuller background text on the objects; the other sources are more descriptive of particular images. Arnett also includes an "Open Issues" section on each planetary body, giving the general reader an idea of major questions that astronomers have not yet answered on the object.

The text is quite readable, statistics check out with standard reference sources, information is updated frequently, and the choices of images are excellent. A significant portion of the images are available only in GIF format, rather than JPG, which requires that larger files be transferred. However, I found response time to be fine from the cited server (several mirror sites are also given)

The less-common animation files require a variety of players, including MPEG, FLI, PICS, and Quicktime, which could be intimidating for some Net users; however, this is the result of the author's extensive knowledge of and links to astronomical resources available on the Internet. Overall, this site is a fun and educational experience for those interested in the heavens and could serve as a reference source for a number of factual inquiries.-Christopher W. Nolan, Trinity University, e-mail: cnolan@ trinity.edu

\section{Feature your collection on the cover of C\&RL News}

CERL News wants to feature aesthetically pleasing photos of items from library collections on its covers. If you have material in your library's collections that you think would make an attractive CERL News cover, please send us color photocopies to review and a brief description of the item and the collection. Selected illustrations will require a color transparency or photograph. Illustrations with a vertical orientation work best. Materials submitted will become the property of CGRL News and cannot be acknowledged. Send to: CERL News Covers, $50 \mathrm{E}$. Huron St., Chicago, IL 60611. 